\title{
Composite nature of fresh skin revealed during compression
}

Benjamin J. Butler MSc

The Royal British Legion Centre for Blast Injury Studies, Imperial College London, London, UK

Surfaces, Microstructure and Fracture Group, Cavendish Laboratory, University of Cambridge, Cambridge, UK

Rachael L. Boddy MSci

Surfaces, Microstructure and Fracture Group, Cavendish Laboratory, University of Cambridge, Cambridge, UK

Chiara Bo PhD

The Royal British Legion Centre for Blast Injury Studies and Institute of Shock Physics, Imperial College London, London, UK Hari Arora PhD

The Royal British Legion Centre for Blast Injury Studies and Department of Bioengineering, Imperial College London, London, UK

Andrew P. Jardine PhD

Surfaces, Microstructure and Fracture Group, Cavendish Laboratory, University of Cambridge, Cambridge, UK

Alun Williams BVMS PhD

Department of Veterinary Medicine, University of Cambridge, Cambridge, UK

William G. Proud PhD

The Royal British Legion Centre for Blast Injury Studies and Institute of Shock Physics, Imperial College London, London, UK

Katherine A. Brown PhD

The Royal British Legion Centre for Blast Injury Studies, Imperial College London, London, UK

Department of Chemistry, The University of Texas at Austin, Austin, Texas, United States

*Corresponding author e-mail address:, kb518@cam.ac.uk

Biological systems are subjected to moderate to high strain rates in blast-type traumatic injuries. An improved understanding of how cells and tissues respond to extreme mechanical stresses could improve mitigation and post-injury treatment strategies. A key aim of our research is to create biologically meaningful injury models of soft tissues. Here we examine the material and cellular properties of freshly harvested porcine skin in compression. Comparative histopathology and analytical modelling suggests that fresh skin differentially responds low to moderate strains rates as a composite rather than that of a homogeneous polymer. The implications of this work are discussed in terms of creating improved analytical models to describe the material behavior of fresh skin.

\section{Introduction}

Blast injury is a common type of injury in modern military combat. ${ }^{1-3}$ Treatment of the wounds in survivors represents a huge clinical challenge. In blast exposure, pressure pulses and strain rate deformation of hard and soft tissues result in injuries and tissue dysfunction uncommon away from the battlefield. ${ }^{4}$ Key contributors to the generation of blast injury include the overpressure of the blast wave in conjunction with its duration (impulse), the environment in which the explosion occurs (free field versus confined space), and the proximity of the target to the explosion. ${ }^{5}$ The levels of force and acceleration experienced in blast injury can be considerable. A stimulus of the duration of $400 \mathrm{~ms}$ with a peak pressure of 600 psi has been estimated to cause $99 \%$ lethality for mammals of $70 \mathrm{~kg}$ weight. ${ }^{6}$ In a more recent analysis of data from more than 2550 large animal blast experiments, new blast injury thresholds for short duration blasts $(<$ $30 \mathrm{~ms}$ ) have been defined to be of 1-2 MPa peak pressures. ${ }^{7}$ Understanding the effects of high strain rates on the material and functional properties of tissues, at lethal and sub-lethal blast conditions, is an important aspect for elucidating the mechanisms associated with damage and repair of wounds from high energy trauma.

Nearly every combat injury involves injury to the skin, generating large amounts of scar tissue in deep skin injuries. ${ }^{8}$ Mammalian skin is a complex, biological material comprised of several layers including an outmost epidermis and a multilayered epithelium. ${ }^{9}$ It functions as a protective barrier for underlying structures. A complex cascade of events is triggered when the integrity of skin is compromised, including hemostasis (cessation of bleeding), inflammation, and wound healing. These events are triggered via mechanisms that can involve both restoration of the epithelium and contracture (which can result in restriction of movement). ${ }^{10}$ The sometimes necessarilylong duration-of treatment for blast injury can lead to poor physiologic and economic outcomes. Specifically, the prolonged break in the continuity of skin, which can arise from many blast injuries, presents a potential portal for infection and can also result in the desiccation of underlying structures. ${ }^{11}$ There is a limited understanding of the material responses of fresh skin as a multi-layered system, particularly at high strains $\left(<1000 \mathrm{~s}^{-1}\right)$. Development of improved material and biological models of the response of fresh skin to traumatic injury has 
great potential to enhance research efforts aimed at mitigating wound damage in combat settings and creating new strategies for improving clinical outcomes of damaged tissues including skin. In this context, we present material and histological data of freshly harvested porcine skin characterized a wide range of strain rates (from $10^{-3}$ to $10^{0}$ ). We relate structural changes observed in optical imaging of skin to its material responses. In addition our data that suggest that treating fresh skin as a homogeneous visco-elastic polymer is insufficient to model its material responses, particularly at higher strain rates. We discuss the implications of our observations and the need for improved models that account for the multilayer properties of skin.

\section{Methods}

Tissue Extraction: Skin samples were obtained from six to eight week old piglets sacrificed by intravenous administration of sodium pentobarbitone. A $6400 \mathrm{~mm}^{2}$ section of skin was excised from the rump of the animal. An $8 \mathrm{~mm}$ diameter biopsy punch was used to isolate full-thickness, circular discs of tissue. The discs were placed in Phosphate Buffered Saline (PBS) solution on ice $\left(4{ }^{\circ} \mathrm{C}\right)$ for the purposes of transport. Prior to testing, the discs were removed from the PBS solution and relevant dimensions (diameter, thickness and weight) were measured. All samples were tested within $10 \mathrm{~h}$ of extraction.

Instron Testing: An Instron 5566 frame fitted with a $2 \mathrm{kN}$ load cell was used to measure the quasi-static response of all biological samples under study. The faces of the load cell and anvil were pre-lubricated and the dried tissue discs were placed unconstrained on the anvil such that the epidermal layer was uppermost. A 'Generic Compression' programme was run which moved the cross-head at a fixed rate until a pre-determined endpoint which corresponded to a sample strain of approximately 0.8 . Utilizing this methodology it was possible to test biological samples at strain rates between $10^{-3} \mathrm{~s}^{-1}$ and $10^{0} \mathrm{~s}^{-1}$. As a comparison, polyurethane-20 (PU-20; Smooth-on, Inc.) circular discs of similar dimensions to tissue samples were cast and cut using the same $8 \mathrm{~mm}$ diameter biopsy punch

Histology: Compressed and uncompressed (control) tissue samples were fixed in $4 \%$ buffered formalin and then prepared for histological analysis. Paraffin wax-embedded sections were cut at a thickness of $4 \mathrm{~mm}$ and stained; Haemotoxylin and Eosin stain were used to evaluate changes in cell and tissue morphology and Masson's Trichrome stain was used to highlight changes to the collagen substructure. Optical data were obtained using a Nikon Eclipse E400 microscope and a JVC camera. 


\section{Material Testing of Porcine Skin}

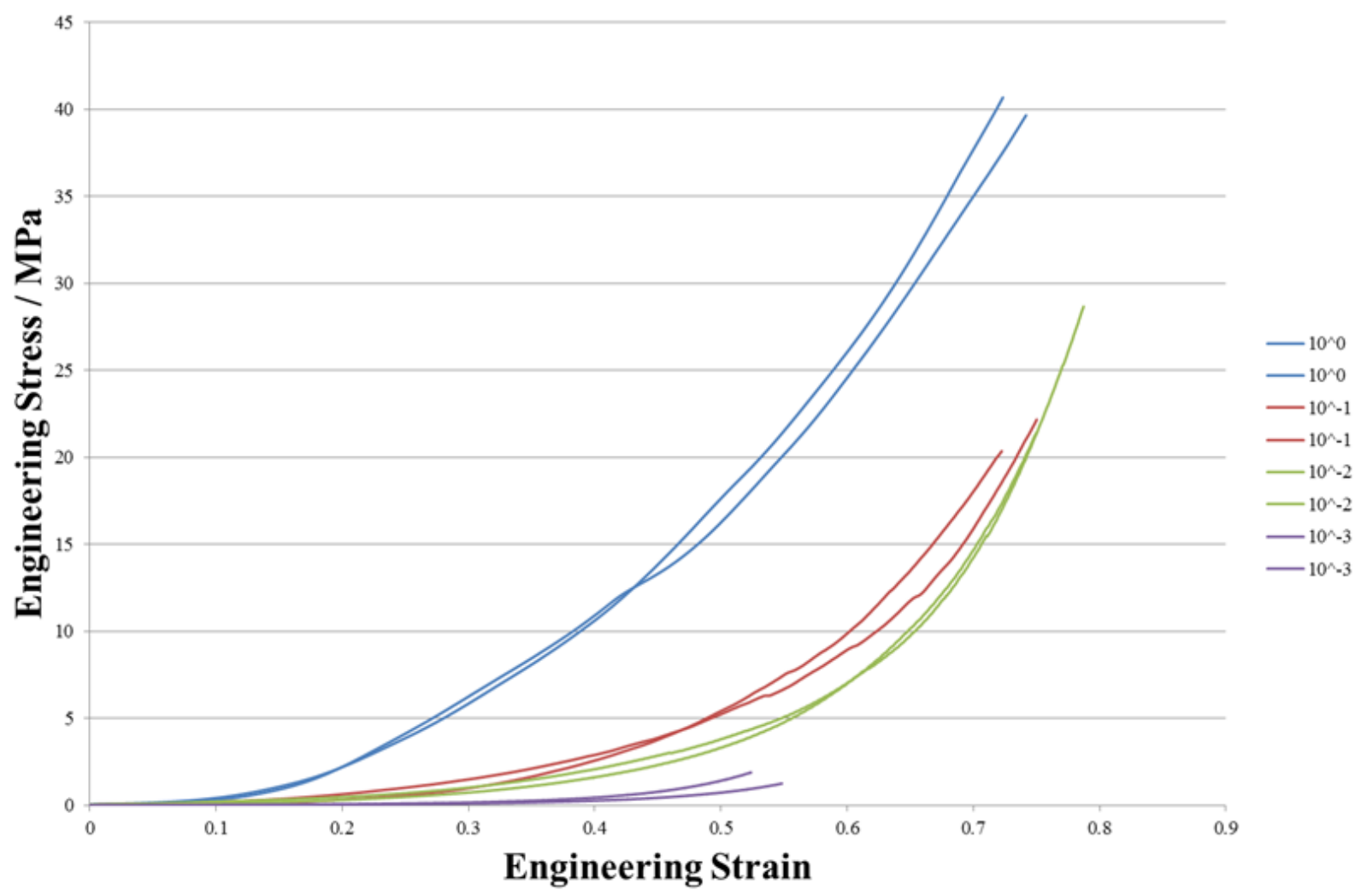

Figure 1. Material response of porcine rump skin samples at varying strain rates. Each line indicates the result of a single sample test, with each colour corresponding to a particular strain rate. The samples tested were excised form a total of three animals and whilst variability between animals and samples can be observed, a trend indicative of strain rate hardening.

The dynamic material response of porcine rump skin samples were investigated over a range of strain rates covering four orders of magnitude $\left(10^{-3} \mathrm{~s}^{-1}\right.$ to $\left.10^{0} \mathrm{~s}^{-1}\right)$. As can be seen in Figure 1, biological samples exhibit variability both sample-to-sample and between animals. However, despite this caveat, strain rate dependent behaviour can be observed indicating hardening of the material at higher strain rates. The extent to which a potential model takes this strain rate effect into account will be the principle determinant of its accuracy. 


\section{Histological Analysis}

To analyse the effect of material testing on the underlying structure of the skin, tested samples were chemically fixed before being stained with dyes commonly used as part of veterinary pathology. An untested sample stained with Haematoxylin and Eosin (H\&E) is shown in Figure 2. H\&E is the 'gold standard' histo-pathological stain which permits visualisation of the tissue structure as a whole, but does not highlight collagen molecules in particular. Porcine skin is a layered structure comprising four general divisions above an underlying layer of subcutaneous fat. Outermost lies keratin, followed by epidermis, papillary dermis and reticular dermis, respectively. While the dermis as a whole is rich in collagen, the density of the collagen fibres present increases with depth.

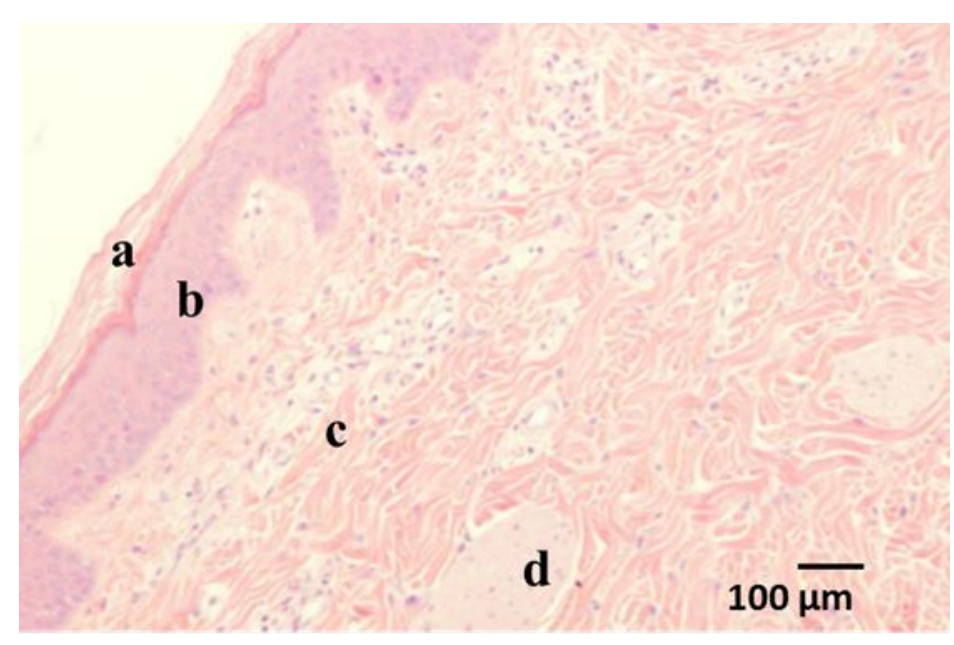

Figure 2. Visual micrograph of an untested, 'sham' sample. The tissue has been stained with H\&E. Cell nuclei appear blue, while the remainder of the tissue takes on a pink hue. The structure of porcine skin can be further subdivided into sections: a) keratin, b) epidermis, c) papillary dermis and d) reticular dermis. The dermis as a whole is rich in collagen fibers, but the density of those fibers increases at greater depths. Scale bar $=100 \mu \mathrm{m}$. 


\section{Strain \\ Rate / s

$\begin{array}{cc}\text { Haematoxylin } & \text { Masson's } \\ \text { \& Eosin } & \text { Trichrome }\end{array}$

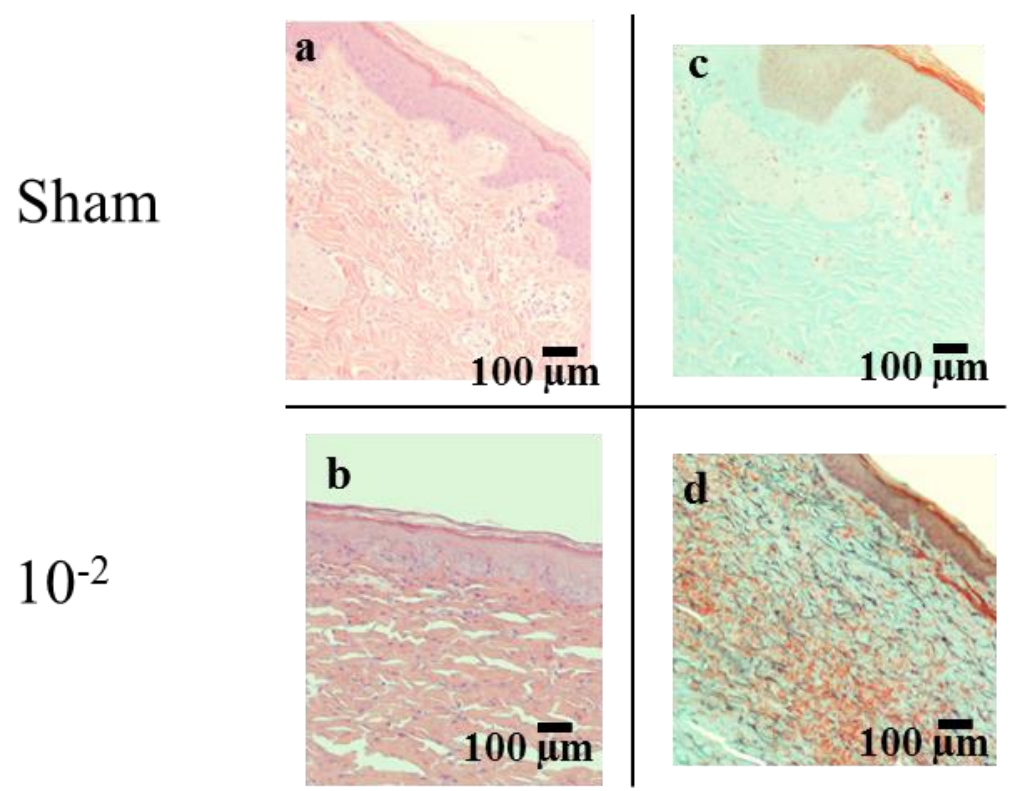

Figure 3. Histological comparison of untested skin and skin tested at a strain rate of $10^{-2}$. Images $\mathbf{a}$ and $\mathbf{b}$ show samples stained with $\mathrm{H} \& \mathrm{E}$ to evaluate changes in cell and tissue morphology, $\mathbf{c}$ and $\mathbf{d}$ are stained with Masson's Trichrome to highlight changes to the collagen substructure. Gross changes brought about by mechanical testing are evident as linear 'voids' present in b, but not visible in a. Orange coloration in images $\mathbf{c}$ and $\mathbf{d}$ is produced by the infiltration of a low molecular weight dye into 'pores' not present in the untested sample. This is possibly due to alterations in the collagen structure not observable in sections stained with H\&E alone. Control, 'sham', samples were excised and transported in an identical manner to the remaining tissue but did not undergo material testing. Scale bars $=100 \mu \mathrm{m}$.

Comparative histological analysis was performed on untested, 'sham' samples and those subjected to mechanical testing, as shown in Figure 3. Changes on the order of tens to hundreds of nanometres were made visible by H\&E staining. At this scale, compressive testing causes the formation of linear voids perpendicular to direction of testing. However, to correlate these changes with alterations at the molecular scale requires the use of other stains, including Masson's trichrome.

Masson's trichrome is a three-part stain comprised of three dyes each of a different molecular weight. The higher molecular weight dyes are capable of displacing the lighter species. The stain, as a whole, therefore, indicates the relative size of any pores present in the sample. In the case of mechanical testing, the lowest weight dye, which produces orange coloration, permeates further into tested samples than negative controls. This suggests that alterations occur on the molecular scale, at the level of collagen molecules, in tandem with those observable in H\&E stained sections. 


\section{Analytical modelling of skin and polymers}

Previously, porcine skin has been treated as a polymer, when analytically describing its mechanical behaviour. For example, number of models have been used to describe the strain rate sensitivity of the material, such as Ogden ${ }^{12-14}$ and other approaches. ${ }^{15,16}$ Shergold $e t$ al. summarise a lot of the previous work conducted on skin characterisation. ${ }^{11}$ Most research in this area, such as Shergold et al., acknowledges the layered nature of skin. However, the study proceeded to capture the mechanical deformation of porcine skin well using homogenised approximations for the material, modelling skin essentially as an isotropic polymer. ${ }^{11}$

It is well known that skin, like any biological material, once removed from its host will begin to degrade. Skin requires specific procedures to maintain freshly harvested tissue near "live" condition prior to testing. In the results presented here, advances in techniques of preparation of biological samples have allowed more detailed rate sensitivities to be observed in the tissue samples. This section will present compressive test data for two sets of material, skin and polyurethane, a material previously described as having intrinsic good mechanical properties that mimic soft tissue ${ }^{18 \text { and references therein }}$. We use van der Waals' hyper-elastic potential in conjunction with a Prony series to describe the nonlinear viscoelastic behaviour of these materials.

The resulting compressive stress-strain responses of the polymers and skin are shown in Figure 4 (a) and Figure 4 (b) respectively for a range of strain rates for porcine skin and polyurethane-20 (PU-20).

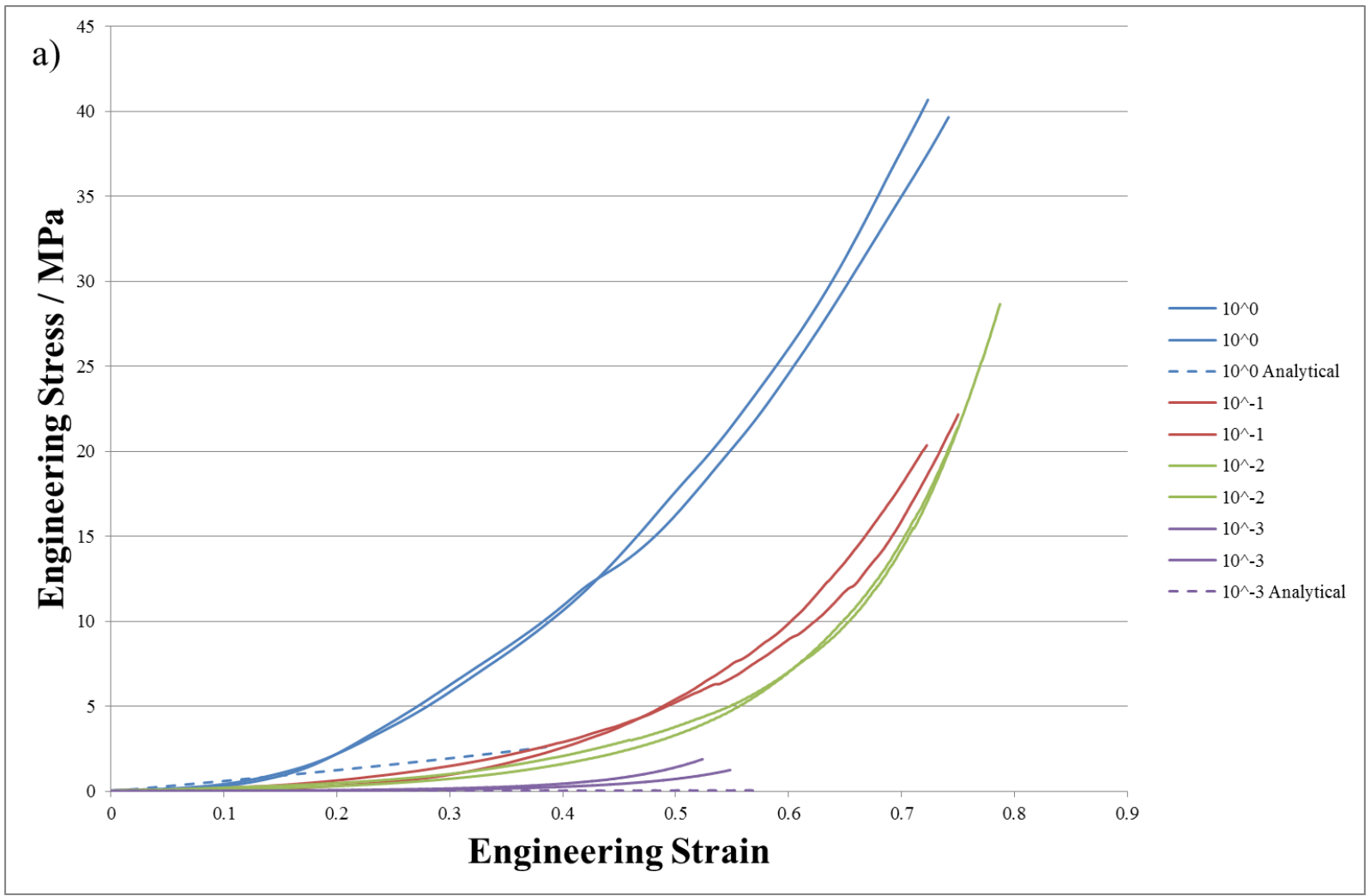




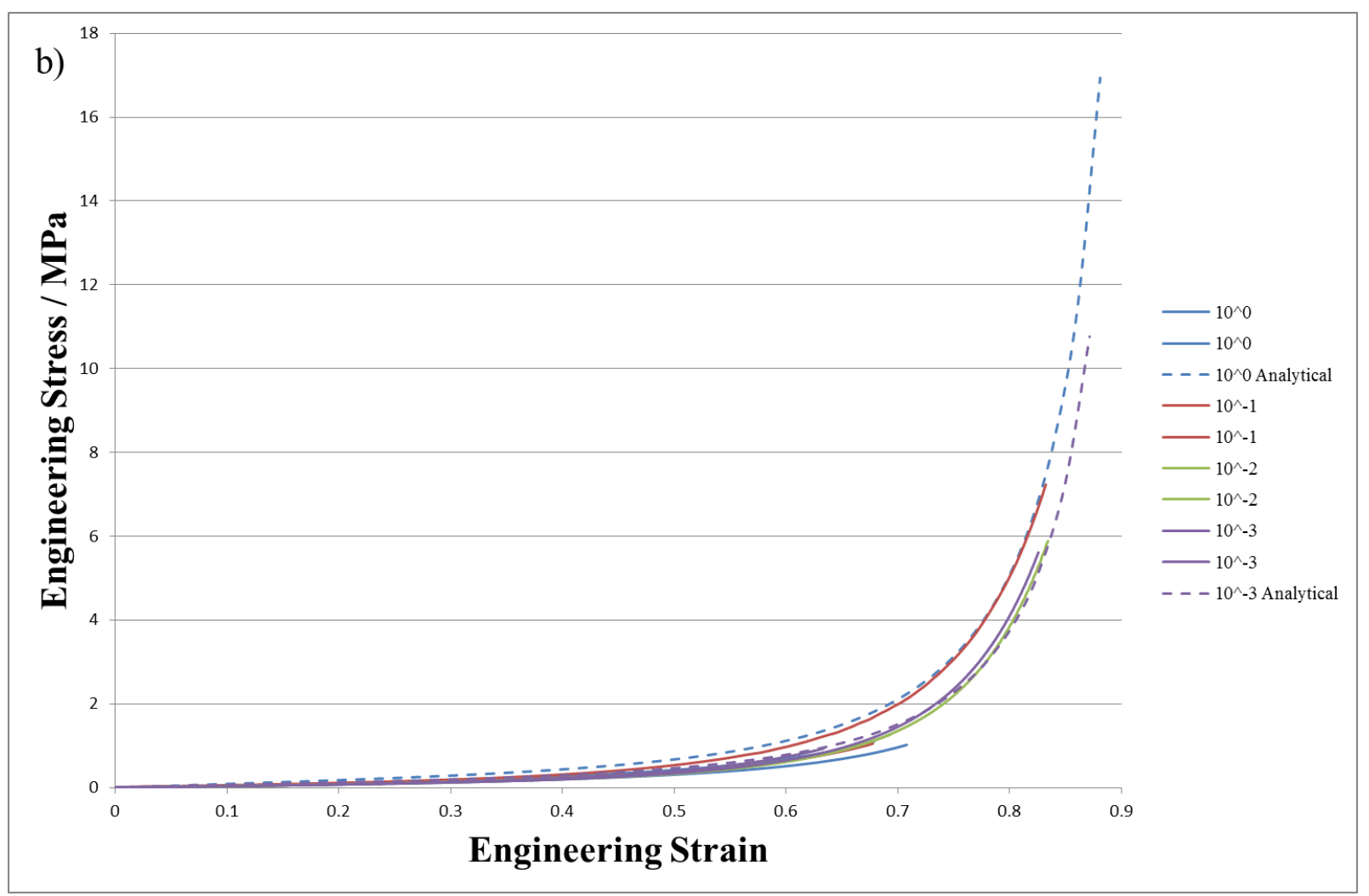

Figure 4. Stress versus strain curves plus analytical fits: (a) Skin; and (b) PU-20. Each line indicates the result of a single sample test or model fit, with each colour corresponding to a particular strain rate. Empirical data is indicated by solid lines whilst analytical fits are represented by dashed lines. In the case of porcine skin, the model is able to capture sample behaviour well at lower strain rates, however, at strain rates approaching $10^{\circ}$, the model and empirical results diverge. The model is superior at capturing the behaviour of polyurethane, though this material exhibits far less strain rate sensitivity.

The graphs showed that PU-20 is a hyper-elastic material and can be represented using the following analytical model. The material's constitutive response under step strain relaxation is both strain and time dependent, where $\sigma$ is the true stress at a true strain $\varepsilon$ and time $t$ :

$$
\sigma(\varepsilon, t)=\sigma_{0}(\varepsilon) g(t)
$$

The strain-dependent function can be non-linear elastic through a hyper-elastic potential. In this work, the van der Waals hyper-elastic potential is used, ${ }^{18}$ which has the following material parameters: a shear modulus, $\psi$, and dimensionless constants, $\lambda_{m}$, the locking-stretch ratio, and $a$, the global interaction parameter. The time-dependent function is represented by the Prony series:

$$
g(t)=g_{\infty}+\sum_{i=1}^{N} g_{i} e^{\left(-t / \tau_{i}\right)}
$$

Where $g_{\infty}$ and $g_{i}$ are dimensionless constants and $g_{\infty}+\Sigma g_{i}=1$. The $\sigma_{0}(\varepsilon)$ term represents the instantaneous stress-strain relationship i.e. at $\mathrm{t}=0$, whilst $\mathrm{g}_{\infty} \sigma_{0}(\varepsilon)$ is the long-term stress-strain relationship, i.e. at $\mathrm{t}=\infty$. The stress for an arbitrary strain history can be expressed in 
the Leaderman form of a convolution integral, ${ }^{19}$ which can then be split into a long term elastic and a viscoelastic contribution, resulting in an expression for stress which is expressed solely in terms of $t$ as shown in Equation 3. If the convolution integral does not have a closed form then the integral can be solved using an algorithm based on finite increments of time. ${ }^{18}$ The van der Waals hyper-elastic potential material constants were determined as were the Prony series constants, with their associated relaxation times in subscript and the resultant curves presented in Figure 4.

$$
\sigma(t)=g_{\infty} \sigma_{0}(t)+\sum_{i=1}^{N} \int_{0}^{t} g_{i} e^{\left(-\frac{t-s}{\tau_{i}}\right)} \frac{d \sigma_{0}(s)}{d s} \cdot d s
$$

It is apparent from Figure 4 (b) that the polymer behavior is captured by this analytical model. One can argue that other simpler models are also suitable for such a polymer. This model was, however, chosen due to its added complexity compared to other material models often chosen in biological tissue modelling. It is clear that although the polymer behavior is adequately enveloped by this form of nonlinear viscoelastic representation, the skin data is not. Initial observations indicate there are significantly greater viscous effects in the biological tissue compared to PU-20. Over a range of three or four orders of magnitude in strain rate the skin data, from $10^{-3} \mathrm{~s}^{-1}$ upwards, the modulus increases four- to five-fold. As the strain rate increases from $10^{-3} \mathrm{~s}^{-1}$ to $10^{3} \mathrm{~s}^{-1}$, a significant strain hardening is observed for the skin samples. This transition cannot adequately be captured using these forms of analytical models.

The reasons for such transitions are suggested by the histology. For the very low strain rates, the deformation is uniform throughout the entire thickness of the dermis layer. The dermis is where the bulk of the damage was observed relative to the tougher epidermal layers. For the very low strain rates, the skin behavior could be captured adequately by the van der Waals' formulation of non-linear viscoelasticity. However, as the strain rate increased, there is a transition in the mode of deformation. Gaps in the dermis were observed in the histological images (Figure 3), most likely arising from disruption of the extracellular matrix proteins such as collagen I. Where there is a transition in deformation or damage mode, there is a need for a new model to describe that behavior. This is why there is a shortcoming at the higher rates, as damage is not uniform and the mode of tissue damage and deformation appears to alter. This leads to significant deviations in the analytical model relative to the experimental data observed in the $10^{0} \mathrm{~s}^{-1}$ curves in Figure 4 (a).

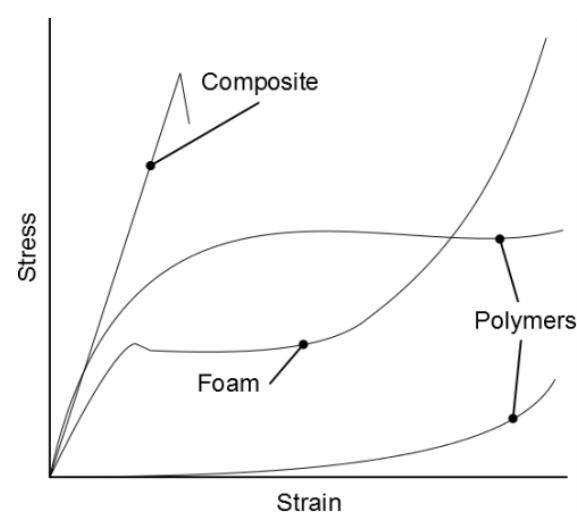

Figure 5. Schematic graph of other material model types, which could be hybridized for skin model applications.

Furthermore, viscoelasticity is generally approached with these analytical forms for small strains. The strains dealt with in blast conditions are large. New approaches that have recently been combined by Del Linz ${ }^{20}$ from various authors ${ }^{21-26}$ have allowed for large strain non-linear viscoelasticity to be more accurately described for polymers. This approach is a step forward in addressing the issues faced here. However there still remains the question of homogeneity. Skin is clearly layered, non-homogeneous composite. Even within a given layer of material, gradients exist, which lead to non-uniform collapse and tearing. The deformations are not elastic. There is permanent deformation of the tissue at various time points during the loading cycle. This contributes to sharp changes in the $\sigma-\varepsilon$ behavior, which are repeatable. A major assumption based within these models is that volume is conserved, but in soft biological materials volume conservation cannot be guaranteed. Therefore it is understandable that these forms of analytical models are not appropriate for representing live or near live tissue. However, averaged approximations of bulk properties can be sufficient depending upon the 
application. The constituent materials within these biological tissues may be described individually using some of these viscoelastic formulations. However the interaction of these materials, in a composite format, is what is required to sensibly and accurately capture the deformation from a bio-mechanical perspective. Figure 5 shows a schematic of other material model types for compression. Aspects of each may be required to capture a different deformation mechanism. For example: micro-buckling of collagen bundles within the extracellular matrix could be captured with a plateau often found in foam structures; the alignment of collagen fibers could be captured a number of ways within viscoelastic/plastic frameworks or densification in porous media. This approach towards a structural or system model over a pure material model seems the most appropriate.

\section{Conclusion}

In this study we have obtained comparative stress-strain curves of fresh skin and a tissue-like polymer using a range of strain rates that yield modest to substantial damage and deformation. A key area of interest for us is to develop material models of soft tissues that are representative of damage processes that can occur in trauma such as blast injury. The data presented here indicates that freshly harvested skin responds as a heterogeneous layered composite material under low to moderate levels of compression. This response is consistent with histopathological images that show non-uniform damage throughout the different layers of skin samples. Comparative modelling of the material behaviour of skin and a tissue-like polymer PU-20 was attempted using a conventional non-linear viscoelastic formulation. The model was able to represent the material properties of PU-20 but was limited in its ability to model fresh skin over the entire range of strains and strain rates applied in these studies. This observation highlights the need to develop more advanced models of soft heterogenous materials, particularly for applications related to understand how soft tissues respond to traumatic injury conditions.

\section{Acknowledgments}

The Centre for Blast Injury Studies acknowledges The Royal British Legion for its support, as well as Imperial College London. The Institute of Shock Physics acknowledges the support of the Atomic Weapon Establishment, Aldermaston, UK and Imperial College London. We thank we thank Madeline Fordham and Louise Webster for their technical expertise in cutting and staining the histopathology samples and Denis Thompson for editing this manuscript.

\section{References}

1. Owens, B. D.; Kragh Jr, J. F.; Macaitis, J.; Svoboda, S. J.; Wenke, J. C. Characterization of extremity wounds in operation Iraqi freedom and operation enduring freedom, J. Orthop. Trauma. 2007, 21, 254-257.

2. Ramasamy, A.; Masouros, S. D.; Newell, N.; Hill, A. M.; Proud, W. G.; Brown, K. A.; Bull, A. M. J.; Clasper, J. C. In-vehicle extremity injuries from improvised explosive devices: current and future foci, Phil. Trans. R. Soc. B. 2011, 366, 160-170.

3. Effgen, G. B.; Hue, C. D.; Vogel, E. $3^{\text {rd }}$; Panzer, M. B.; Meaney, D. F.; Bass, C. R.; Morrison, B. $3^{\text {rd }}$. A Multiscale Approach to Blast Neurotrauma Modeling: Part II: Methodology for Inducing Blast Injury to in vitro Models. Front. Neurol. $2012,3,23$.

4. Ramasamy, A.; Hill, A. M.; Masouros, S. D.; Gibb, I.; Bull, A. M. J.; Clasper, J. C. Blast-related fracture patterns: a forensic biomechanical approach, J. R. Soc. Interface. 2011, 8, 689-698.

5. Proud, W. G. The physical basis of explosion and blast injury processes. J. R. Army Med. Corps. 2013, 159, Suppl 1:i4-i9.

6. Richmond, D. R.; White, C. S. A Tentative Estimation of Mans Tolerance to Overpressures from Air Blast, The Symposium on Effectiveness Analysis Techniques for Non-Nuclear Warheads Against Surface Targets, Dahlgren, Virginia, 1962.

7. Bass, C. R.; Rafaels, K. A.; Salzar, R. S. Pulmonary injury risk assessment for short-duration blasts, J. Trauma. 2008, 65, 604-615.

8. Christopherson, G. T.; Nesti, L. J. Stem cell applications in military medicine, Stem Cell Res. Ther. 2011, 2(5), 40.

9. Shimizu H. 2007. Shimizu's Textbook of Dermatology. Hokaido University Press, Japan.

10. Watt, A. J.; Friedrich, J. B.; Huang, J. I. Advances in treating skin defects of the hand: skin substitutes and negative-pressure wound therapy, Hand Clin. 2012, 28(4), 519-528.

11. Shergold, O. A.; Fleck, N. A.; Radford, D. The uniaxial stress versus strain response of pig skin and silicone rubber at low and high strain rates, Int. J. Impact Engineering. 2006, 32, 1384-1402.

12. Lim, J.; Hong, J.; Chen, W. W.; Weerasooriya, T. Mechanical response of pig skin under dynamic tensile loading, Int. J. Impact Engineering. 2011, 38, 130-135.

13. Wu, J. Z.; Dong, R. G.; Smutz, P.; Schopper, A. W. Nonlinear and viscoelastic characteristics of skin under compression: experiment and analysis, Bio-medical Materials and Engineering. 2003, 13, 373-385.

14. Crichton, M. L.; Donose, B. C.; Chen, X.; Raphael, A. P.; Huang, H.; Kendall, M. A. The viscoelastic, hyperelastic and scale dependent behaviour of freshly excised individual skin layers, Biomaterials. 2011, 20, 4670-4681. 
15. Groves, R. B.; Coulman, S. A.; Birchall, J. C., Evans, S. L. An anisotropic, hyperelastic model for skin: experimental measurements, finite element modelling and identification of parameters for human and murine skin, J. Mech. Behav. Biomed. Mater. 2013, 18, 167-180.

16. Flynn, C.; Rubin, M. B. An anisotropic discrete fibre model based on a generalised strain invariant with application to soft biological tissues, Engineering Science. 2012, 60, 68-76.

17. Soldani, G, Losi, P., Milioni, C. and Raffi, A. Light microscopy evaluation of polyurethane vascular grafts porosity by Sudan Black B staining, Journal of Microscopy, 206, 2002, 139-145

18. Goh, S. M.; Charalambides, M. N.; Williams, J. G. Determination of the Constitutive Constants of Non-Linear Viscoelastic Materials, Mechanics of Time-Dependent Materials, 2004, 8, 255-268.

19. Williams, J. G. Stress Analysis of Polymers, 2nd edn. Hoboken, NJ: John Wiley \& Sons, 1980.

20. Del Linz, P. PhD Thesis, Imperial College London, 2014.

21. Lubliner, J.; Plasticity Theory, London: Macmillan Publishing, 1980.

22. Arruda, E. M.; Boyce, M. C. A three-dimensional constitutive model for the large stretch behavior of rubber elastic materials, Mechanics and Physics of Solids. 1993, 41, 389-412.

23. Huber, N.; Tsakmakis, C. Finite deformation viscoelasticity laws, Mechanics of Materials. 2000, 32, 1-18.

24. Amin, A.; Alam, M. S.; Okui, Y. An improved hyperelasticity relation in modeling viscoelasticity response of natural and high damping rubbers in compression: experiments, parameter identification and numerical verification, Mechanics of Materials. 2002, 34, 75-95.

25. Amin, A.; Lion, A.; Sekita, S.; Okui, Y. Nonlinear dependence of viscosity in modeling the rate-dependent response of natural and high damping rubbers in compression and shear: Experimental identification and numerical verification, Int. J. Plasticity. 2006, 22, 1610-1657.

26. Hoo Fatt, M. S.; Ouyang, X. Three-dimensional constitutive equations for Styrene Butadiene Rubber at high strain rates, Mechanics of Materials. 2008, 40, 1-16. 